\title{
Standard hydrogen electrode and potential of zero charge in density functional calculations
}

Tripkovic, Vladimir; Björketun, Mårten; Skúlason, Egill; Rossmeisl, Jan

Published in:

Physical Review B Condensed Matter

Link to article, DOI:

10.1103/PhysRevB.84.115452

Publication date:

2011

Document Version

Publisher's PDF, also known as Version of record

Link back to DTU Orbit

Citation (APA):

Tripkovic, V., Björketun, M., Skúlason, E., \& Rossmeisl, J. (2011). Standard hydrogen electrode and potential of zero charge in density functional calculations. Physical Review B Condensed Matter, 84(11), 115452.

https://doi.org/10.1103/PhysRevB.84.115452

\section{General rights}

Copyright and moral rights for the publications made accessible in the public portal are retained by the authors and/or other copyright owners and it is a condition of accessing publications that users recognise and abide by the legal requirements associated with these rights.

- Users may download and print one copy of any publication from the public portal for the purpose of private study or research.

- You may not further distribute the material or use it for any profit-making activity or commercial gain

- You may freely distribute the URL identifying the publication in the public portal 


\title{
Standard hydrogen electrode and potential of zero charge in density functional calculations
}

\author{
Vladimir Tripkovic, ${ }^{1}$ Mårten E. Björketun, ${ }^{1}$ Egill Skúlason, ${ }^{2}$ and Jan Rossmeisl ${ }^{1, *}$ \\ ${ }^{1}$ Center for Atomic-scale Materials Design, Department of Physics, Technical University of Denmark, DK-2800 Lyngby, Denmark \\ ${ }^{2}$ Science Institute, VR-III, University of Iceland, IS-107 Reykjavik, Iceland
}

(Received 25 May 2011; published 26 September 2011)

\begin{abstract}
Methods to explicitly account for half-cell electrode potentials have recently appeared within the framework of density functional theory. The potential of the electrode relative to the standard hydrogen electrode is typically determined by subtracting the experimental value of the absolute standard hydrogen electrode potential (ASHEP) from the calculated work function. Although conceptually correct, this procedure introduces two sources of errors: (i) the experimental estimate of the ASHEP varies from 4.28 to $4.85 \mathrm{~V}$ and, as has been previously shown and is reconfirmed here, (ii) the calculated work function strongly depends on the structure of the water film covering the metal surface. In this paper, we first identify the most accurate experimental reference for the ASHEP by revisiting up-to-date literature, and validate the choice of electron reference level in single-electrode density functional setups. By analyzing a dozen different water structures, built up from water hexamers, in their uncharged [potential of zero charge (PZC)] states on $\mathrm{Pt}(111)$, we then determine three different criteria (no net dipole, no charge transfer, and high water flexibility) that a water structure should possess in order for its computed ASHEP to closely match the experimental benchmark. We capture and quantify these three effects by calculating trends in the ASHEP and PZC on eight close-packed transition metals, considering the four most simple and representative water models. Finally, it is also demonstrated how the work function changes with exchange-correlation functional.
\end{abstract}

DOI: 10.1103/PhysRevB.84.115452

PACS number(s): 68.08.-p, 73.30.+y, 71.15.Nc, 71.15.Mb

\section{INTRODUCTION}

In electrochemistry, all half-cell electrode potentials are given relative to a chosen reference electrode of some wellknown reaction, for example, the standard calomel or the standard hydrogen electrode (SHE). In many electrochemistry experiments, taking the difference between two electrode potentials alleviates the problem of determining the potential on an absolute scale. Nevertheless, the absolute potential scale is still of great importance when comparing electrochemical and ultrahigh vacuum (UHV) experiments and when trying to match semiconductor and solution energy levels ${ }^{1}$ in, for instance, photoelectrochemical devices.

Density functional theory (DFT) based methods for modeling electrochemical systems and, in particular, for treating charge-transfer reactions at the electrochemical interface have just started to appear. ${ }^{2-12}$ In these simulations, the electrochemical cell is customarily split into two half-cells and the redox reactions taking place at the two electrodes are studied separately. Here, the absolute potential enters as a key parameter because it is no longer possible to measure potential differences between the two electrodes. We would, however, like to distinguish between three different categories of theoretical electrochemistry studies. In the first category, we find investigations of the thermodynamics of electrochemical reactions and calculations of redox potentials. ${ }^{13,14}$ In these studies, the potential does not have to be taken explicitly into account in the DFT simulation, but can rather be added a posteriori. The second category comprises the energetics of charge-transfer reactions. To study these types of problems, it is necessary to explicitly include the potential in the DFT model. At the same time, it is possible to introduce an internal potential reference, which means the accuracy of the absolute potential is of less importance. ${ }^{15,16}$ Finally, in the third category, we find problems like the matching of semiconductor and solution energy levels that demand highly accurate estimates of the absolute potential.

In general, the DFT based methods use the work function (WF) of the water-covered metal electrode as a measure of the absolute electrode potential. In order to obtain a relative potential scale, the WF scale needs to be coupled to a normal thermoelectrochemical scale, for instance, of the SHE. This matter can be approached in two conceptually different ways. The most straightforward and most common is to use an experimental value of the absolute standard hydrogen electrode potential (ASHEP), i.e., the experimental WF that corresponds to SHE conditions, as reference. However, there is a large uncertainty in the ASHEP reported in the literature; they vary from 4.28 to $4.85 \mathrm{~V} \cdot{ }^{17-26}$ Many properties, including energy barriers and, hence, rates of charge-transfer reactions, are strongly potential dependent, which renders the use of the most correct experimental estimate essential. Moreover, since the calculated WF depends strongly on the structure of the water at the metal-aqueous interface, ${ }^{27,28}$ employing an experimental reference is a sound approach only as long as the real system is faithfully mimicked in the simulations. It should be noted, though, that it is usually difficult to capture properties of bulk water in these types of first-principles simulations as computational constraints limit the number of water molecules that can be explicitly treated. ${ }^{6,29}$ Alternatively, one can use a theoretical value of the ASHEP, internal to the system and perhaps different for different metal-water-vacuum setups. This approach has the advantage that it requires a less realistic representation of the simulated metal-water interface, but in turn a scheme for determining the theoretical ASHEP is needed. We have recently devised such a scheme ${ }^{15}$ and, subsequently, successfully implemented it to model the hydrogen evolution and oxidation reactions. ${ }^{16}$ 
Although, as we have already discussed, many problems can be solved using an internal "low-quality" ASHEP reference, there are cases such as the previously mentioned matching of energy levels when a good theoretical estimate of the ASHEP is vital. Therefore, in this paper, we present an extensive investigation of the theoretical ASHEP. Particular attention is given to how the choice of water model, used to emulate the interfacial water, affects the calculated ASHEP and makes it deviate from the experimental counterpart. Much effort is also put into evaluating the somehow related quantity potential of zero charge (PZC). By thoroughly examining the available literature, we first determine the most reliable experimental benchmark value for the ASHEP. Invoking the fundamental concepts of the absolute potential scale, developed by Trasatti and others, ${ }^{1,30-35}$ we then pinpoint the most appropriate choice for the reference level of the computed WF. Subsequently, by briefly revisiting our earlier works, we show how an internal ASHEP reference can be readily established. ${ }^{15,16}$ Next, we perform a comprehensive investigation of $\mathrm{Pt}(111)$-water systems. We demonstrate how the WF, which corresponds to the PZC when the water film contains no ions, varies with the structure of the interfacial water and identify the different contributions to the WF. Guided partly by this study, we identify three properties that the model water film must possess in order to reproduce the experimental ASHEP. We then evaluate the ASHEP for a set of representative electrode-water systems and quantify the deviations from the experimental value owing to the use of unrealistic water structures.

\section{EXPERIMENTAL ESTIMATES OF THE ASHEP}

Experimental values of the ASHEP reported in the earlier literature resided mostly on measurements of changes in the WF upon water adsorption. The WF was measured by means of immersed electrode setups, ${ }^{20-22}$ UHV studies, ${ }^{36,37}$ or, similarly, through measurements of the potential difference across the $\mathrm{Hg} \mid$ air $\left|\mathrm{H}^{+}\right| \mathrm{Pt}, \mathrm{H}_{2}$ cell in the absence of any specific adsorption or charge transfer (so-called streaming $\mathrm{Hg}$ jet method). ${ }^{17,18}$ There is a large scatter in reported values obtained using these techniques (4.44 to $4.85 \mathrm{~V}$ ). The discrepancies have been attributed to, among other factors, surface contamination, the orientation of water in UHV experiments being different from the one in bulk water, and to partial charge transfer. ${ }^{30,32}$ Here, it is worth noting that the lower value has been identified as the most reliable one by Trasatti and also the value recommended by the International Union of Pure and Applied Chemistry (IUPAC). ${ }^{1}$

A second and more direct approach to measure the ASHEP is through the Born-Haber cycle shown in Fig. 1, where $\Delta G_{\mathrm{d}}$, $\Delta G_{\mathrm{I}}, \alpha_{S}\left(\mathrm{H}^{+}\right)$, and $\mu_{\mathrm{e}^{-}}$are dissociation and ionization free energies, the real potential of the proton in solution, and the chemical potential of the electron with respect to its reference state (a measure of the ASHEP). As indicated in Fig. 1, $\mathrm{H}^{+}(S)+\mathrm{e}^{-}(M)$ will be in equilibrium with $\frac{1}{2} \mathrm{H}_{2}(g)$ at the $\mathrm{SHE}$ potential under standard conditions $\left(\mathrm{pH}=0, p_{\mathrm{H}_{2}}=1\right.$ bar, $T=298 \mathrm{~K})$. Since the free energies of $\mathrm{H}_{2}(g)$ and $\mathrm{H}^{+}(S)$ are independent of the metal used as electrode as long as $\mathrm{H}^{+}$is solvated in the bulk of the solution, the equilibrium of the (a)

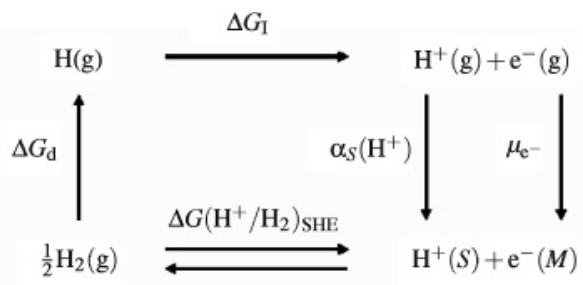

(b)

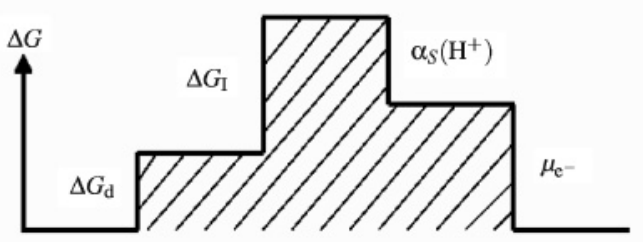

FIG. 1. (a) Born-Haber cycle for the standard hydrogen electrode. (b) Energies in (a) shown schematically on a step diagram. The ASHEP is equal to $\mu_{\mathrm{e}^{-}}$.

reaction

$$
\mathrm{H}^{+}(S)+\mathrm{e}^{-}(M) \leftrightarrow \frac{1}{2} \mathrm{H}_{2}(g)
$$

uniquely defines the Fermi level $E_{\mathrm{F}}(M)$ (which makes it metal independent). In other words, the amount of charge transferred on different metal surfaces will always be such that the metal Fermi levels will become aligned at SHE conditions.

The ASHEP can thus be determined directly from the Born-Haber cycle, without involving the WF, if the proton solvation energy is accurately measured. Recently, through very precise determination of $\alpha_{S}\left(\mathrm{H}^{+}\right)$, the ASHEP has been estimated to be $4.42 \mathrm{~V}^{25,38}$ This value is very close to the $4.44 \mathrm{~V}$ recommended by Trasatti. In fact, it is highly unlikely that this striking agreement between two completely different approaches can be the result of a pure coincidence. It should be noted, however, that in an even more recent study, a slightly lower value, ASHEP $=4.28 \mathrm{~V}$, was suggested. ${ }^{26}$ This value was obtained by using the electron in vacuum at $0 \mathrm{~K}$ as the zero level for the electron energy and the standard hydration free energy of the proton $\Delta G_{S}^{0}\left(\mathrm{H}^{+}\right)$instead of $\alpha_{S}\left(\mathrm{H}^{+}\right)$. Nevertheless, as we shall see in the next section, the most natural and convenient choice of zero level for the electron energy in our DFT setup is the electron in vacuum close to the solution surface, which is also the zero level assumed by IUPAC. ${ }^{1}$ In this work, we will therefore use $4.44 \mathrm{~V}$ as the experimental value to benchmark our calculated ASHEPs against.

\section{FREE-ELECTRON REFERENCE IN EXPERIMENTS AND DFT}

As Trasatti has pointed out, the ASHEP depends on the chosen reference state for an electron at rest. ${ }^{1,30,33}$ He has showed that there exist three such physically conceivable levels: at rest in vacuum at infinity, in the bulk of the solution, or in vacuum close to the surface of the solution. From his analysis, he concluded that the best reference is the nearsolution-surface vacuum because it establishes a direct link between surface science and electrochemical experiments and, furthermore, it is amenable to experimental determination. ${ }^{1,33}$ 

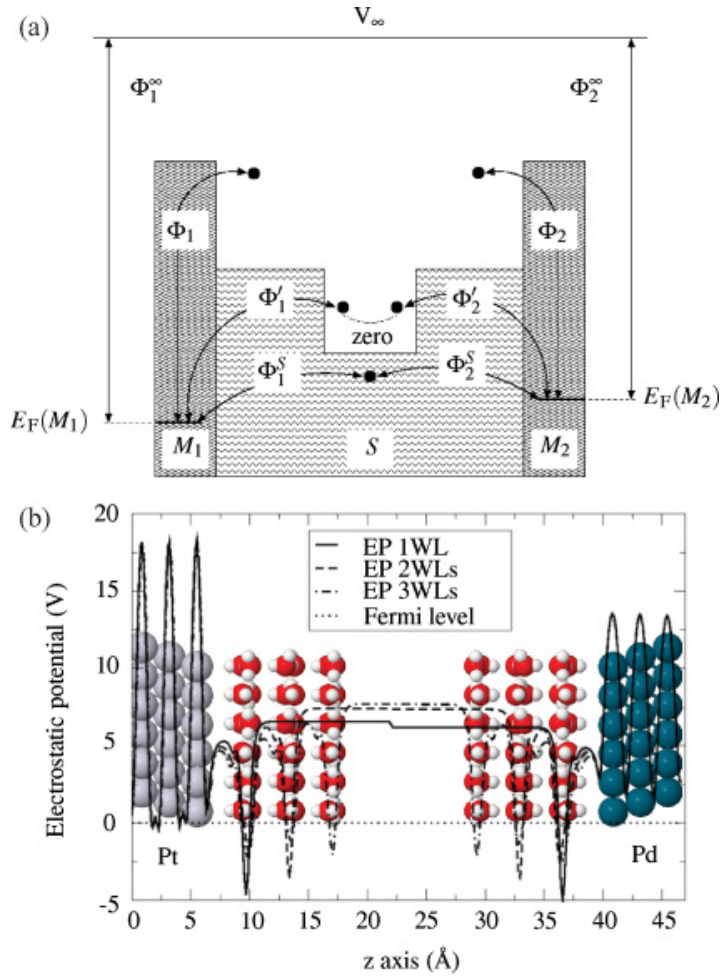

FIG. 2. (Color online) (a) Schematics of an electrochemical cell showing a set of work functions $\left\{\Phi_{i}^{k}\right\}$ measuring the work needed to bring an electron from the Fermi level of the metal to four different free-electron reference states. $\Phi_{i}^{\infty}, \Phi_{i}^{S}, \Phi_{i}$, and $\Phi_{i}^{\prime}$ denote infinite vacuum, bulk solution, near vacuum, and near-solution-surface vacuum WFs. (b) DFT model of an electrochemical cell comprised of $\mathrm{Pt}(111)$ and $\mathrm{Pd}(111)$ electrodes immersed in a common ion-free aqueous solution, vacuum cleaved half-way between the electrodes. The $z$ axis equals the unit-cell size in the surface normal direction. The figure illustrates the convergence of the electrostatic potential with respect to the number of water layers. The water layers are mirror imaged on the two electrodes.

As we shall see in the following, it is also the most natural reference in DFT calculations.

A typical electrochemical cell is illustrated in Fig. 2(a). Appropriate half-cells are obtained when the electrochemical cell is vacuum cleaved at a point in the solution where the electrostatic potential (EP) is no longer affected by the metal surface. Four WFs required to bring an electron from the Fermi level of the metal $M_{i}$ to a chosen reference state are indicated, where labels $\Phi_{i}^{\infty}, \Phi_{i}^{S}, \Phi_{i}$, and $\Phi_{i}^{\prime}$ stand for far (or infinite) vacuum, bulk solution, near vacuum, and near-solution-surface vacuum WFs. Three of them, $\Phi_{i}^{\infty}, \Phi_{i}^{S}$, and $\Phi_{i}^{\prime}$, are potentially relevant for determination of the ASHEP.

In conventional periodic DFT calculations, $\Phi_{i}^{\infty}$ is not defined because of infinite surfaces imposed by periodic boundary conditions. As a consequence of the infinite extension of the surface, even a point in vacuum infinitely far from the surface will feel the presence of the surface dipoles. The second plausible reference is the point in bulk solution. Obtaining a solution reference point is straightforward given that enough water is included in the cell. This, however, reflects on the system size, and the computational cost needed to perform such a relaxation is staggering. Additionally, a portion of the water must be fixed because any wiggling of the water molecules can shift the reference level. ${ }^{6}$ In contrast, the near-solution-surface vacuum level can be readily obtained by the WF in metal-water-vacuum setups and, moreover, the measurement does not entail any confinements. Hence, it is the most natural free-electron reference state in DFT calculations, and $\Phi_{i}^{\prime}$ is thus the most suitable WF.

The DFT counterpart to an electrochemical cell is shown in Fig. 2(b). In ordinary DFT simulations of such setups, the Fermi levels of the two electrodes $\left(M_{1}\right.$ and $\left.M_{2}\right)$ will always be aligned irrespective of the absolute potential and the electrode material. Once the Fermi levels of $M_{1}$ and $M_{2}$ are aligned, $\Phi_{1}^{\prime}$ and $\Phi_{2}^{\prime}$ should also become equal (since alignment of the Fermi levels means that the electrodes have assumed the same potential), given that the solution phase is thick and polarizable enough to screen the fields from the metals. However, this is usually not the case in DFT calculations due to the limited amount of water that one can afford to include in the simulations. Convergence of the EP profile for a $\mathrm{Pt}(111) \mid$ water|vacuum $\mid$ water $\mid \operatorname{Pd}(111)$ cell with respect to the number of water layers is displayed in Fig. 2(b). A gradual improvement of the near-solution-surface reference point with increasing thickness of the water is clearly observed. The mid-vacuum EP discontinuity (obtained by electrostatic decoupling of periodically repeated supercells) is fairly large in the cell containing only a single water layer. However, it becomes much smaller after addition of a second layer and it essentially vanishes when a third water layer has been added. In Sec. V, we will demonstrate how inadequate water structures introduce, sometimes substantial, errors in the calculated ASHEP and PZC. It turns out that efficient screening is one of the keys to accurately predicting these properties.

\section{ESTABLISHING A THEORETICAL, INTERNAL REFERENCE FOR THE ASHEP}

In this section, we describe how an internal reference for the ASHEP can be established in a DFT based electrochemistry study. The methodology is summarized here because of its relevance for the following study. For more details about the procedure, we refer to our previous works. ${ }^{15,16}$

We start with an atomic setup consisting of a metal slab and an electrolyte represented by water layers outside the surface [cf. Fig. 2(b)]. The metal-electrolyte interface is charged by adding hydrogen atoms to the first water layer outside the metal surface. These hydrogen atoms spontaneously separate into protons that become solvated in the water bilayer and electrons that are transferred to the surface of the metal slab. The charge separation, in turn, creates an EP drop across the interface. The surface charge, and hence the potential, can be varied in steps by changing the concentration of protons in the water layer.

A link between the thermoelectrochemical scale of the SHE and the WF $\Phi^{\prime}$ can be established by focusing on the free energy of the solid-liquid interface as it is charged with protons and electrons. The total or integral free energy per surface metal atom (or surface area) relative to $\mathrm{H}_{2}$ for a system with $n$ 
protons and $N$ surface atoms is given by

$$
G_{\text {int }}=\left(G(N, n)-G(N, 0)-\frac{n}{2} \mu_{\mathrm{H}_{2}}\right) / N,
$$

where $\mu_{\mathrm{H}_{2}}$ is the reference chemical potential of hydrogen. $G_{\text {int }}$ corresponds to the free energy stored in the interface set up by the protons in the water layer and their counter charge in the metal. $G_{\text {int }}$ will be quadratic in potential if the interface behaves as a perfect capacitor. The derivative of $G_{\text {int }}$ with respect to the proton concentration is the chemical potential of protons and electrons

$$
\frac{d G_{\mathrm{int}}}{d(n / N)}=\mu_{\left(\mathrm{H}^{+}+\mathrm{e}^{-}\right)}-\frac{1}{2} \mu_{\mathrm{H}_{2}} .
$$

The role of the $\mu_{\mathrm{H}_{2}}$ term is to define the reference. Hence, if we choose $\mu_{\mathrm{H}_{2}}$ to equal the free energy of $\mathrm{H}_{2}(g)$ at standard conditions, the WF corresponding to the minimum of $G_{\text {int }}$ will define the potential of the SHE (reaction (1) is in equilibrium) on an absolute scale.

\section{RESULTS AND DISCUSSION}

In the following, we first (Sec. V A) calculate the PZC of $\mathrm{Pt}(111)$ using a large number of different water models and both Perdew-Burke-Ernzerhof (PBE) and revised Perdew-Burke-Ernzerhof (RPBE) exchange-correlation (XC) functionals (for computational details, see Appendix A). We define the PZC as the WF $\Phi_{\mathrm{Pt}(111)}^{\prime}$ of the metal covered with an ion-free (i.e., in our case unprotonated) water film. ${ }^{1,31}$ The calculated PZC is found to depend strongly on the structure of the water film, and the choice of XC functional turns out to be of importance too. We disclose the physical origin of the large scatter in calculated values and discuss its consequences. Subsequently (Sec. V B), the ASHEP and PZC are computed on the most close-packed surfaces of eight transition metals $M=\{\mathrm{Ru}, \mathrm{Pd}, \mathrm{Pt}, \mathrm{Au}, \mathrm{Ag}, \mathrm{Re}, \mathrm{Rh}, \mathrm{Ir}\}$, using four qualitatively different water structures. These results are then used to discern metal- (or WF-) dependent trends in the PZC and to illustrate the importance of a set of physical properties of the water film for accurate estimation of the ASHEP. Finally, in Sec. V C, we comment briefly on the use and applicability of external (universal) and internal ASHEP references.

Before presenting the PZC and ASHEP results, we will briefly introduce the water models used in this study (more specific details are given in the subsequent sections). The water films are formed from bilayers of different net dipole orientations, stacked in a layer-by-layer fashion. From UHV and DFT studies, it has been inferred that, on many close-packed transition-metal surfaces such as $\mathrm{Pt}(111),{ }^{39-44} \mathrm{Ru}(0001),{ }^{44-46}$ $\mathrm{Pd}(111),{ }^{44,47}$ and $\mathrm{Rh}(111),{ }^{44,48}$ the low-temperature structure consists of water molecules adsorbed in the form of hexagonal rings, which give rise to the well-known honeycomb pattern. Furthermore, it has been shown that the diversity of the hexagon-based structures is rich. For example, the layer forming on $\mathrm{Ru}(0001)$ is half dissociated ${ }^{45}$ and triangular depressions are found in the layer forming on $\mathrm{Pt}(111) .{ }^{49}$ The hexagonally structured water layer has been named bilayer structure because it consists of two differently oriented water molecules, located at slightly different distances from the surface; one is lying flat with the molecular dipole plane nearly parallel to the surface, and the other one has a dangling hydrogen bond directed either away or toward the surface. To distinguish between these two orientations, we will henceforth refer to them as H-up and H-down structures. We note that these structures have previously been successfully employed in modeling of hydrogen oxidation-evolution and oxygen reduction reactions. ${ }^{16,50}$

Although there is still little experimental evidence as to whether these structures will be preserved at ambient conditions, a recent molecular dynamics study showed that a mixture of $\mathrm{H}$-up and $\mathrm{H}$-down structures is likely to exist at $T=300 \mathrm{~K}$ on $\mathrm{Pt}(111)$ and $\mathrm{Ru}(0001) .{ }^{27}$ Albeit in the same study, the authors found that, on some other close-packed transition-metal surfaces ( $\mathrm{Au}, \mathrm{Ag}$, and $\mathrm{Pd}$ ), water molecules acquire random orientations and the hexagonal pattern becomes disrupted. These metals will also be investigated in this work, but since our aim is to systematically study how the PZC and ASHEP vary with water model rather than to identify the most stable water structure under a certain set of environmental conditions, the uncertainty about the actual structure will not affect our conclusions.

\section{A. Sensitivity of the PZC to water structure and XC functional}

In this section, we carry out a detailed analysis of the PZC of $\mathrm{Pt}(111)$ covered with thin water films. The PZC is evaluated for water films of single- and double-bilayer thickness for almost all conceivable combinations of H-up, H-down, and H-mixed (a neutral layer containing an equal number of molecular dipoles pointing up and down) bilayers. Additionally, we look at thicker films, containing up to five H-mixed layers. Some PZC data for the other seven transition metals will be presented in Sec. V B. The results for $\mathrm{Pt}(111)$ are summarized in Table I. The systems are grouped according to the nominal net dipole moment ( $D_{\mathrm{WL}}=x \mathrm{~d}$ ) of the adsorbed water film (we use the convention that $x>0$ for net dipoles pointing away from the metal surface, $x<0$ for net dipoles pointing toward the surface, and $x=0$ for neutral films). Schematics of the films are shown in the first column of the table, with arrows indicating the dipole moments of individual water layers. Two arrows pointing in the same direction indicate a finite dipole moment, whereas oppositely aligned arrows indicate a nominally neutral water layer that is expected to have no net dipole.

According to DFT calculations with the RPBE (PBE) XC functional, the WF $\Phi_{\mathrm{Pt}(111)}$ of pristine $\mathrm{Pt}(111)$ is $5.60(5.74) \mathrm{eV}$. As a comparison, the experimental value for $\mathrm{Pt}(111)$ currently given in Ref. 51 is $5.93 \mathrm{eV}$. Upon adsorption of a water film, the metal WF changes by $\Delta \Phi=\Phi_{\mathrm{Pt}(111)}^{\prime}-\Phi_{\mathrm{Pt}(111)}$. The magnitude and sign of $\Delta \Phi$ depend on the structure of the water film and the distance between the metal surface and the first water bilayer. As shown by Materzanini et al. and by Jinnouchi and Anderson, $\Delta \Phi$ can, to a good approximation, be divided into two distinct contributions. ${ }^{11,52}$ The first, $\Delta \Phi_{\text {orient }}$, stems from the orientation of the water molecules and is obtained by calculating the dipole moment of the water film, isolated and frozen in the geometry it adopts at the metal surface. The second contribution, the polarization part $\Delta \Phi_{\text {polar }}$, is caused by charge redistribution at the metal-water interface upon adsorption of the water film. Most of the charge transfer is 
TABLE I. Work function $\Phi_{\mathrm{Pt}(111)}^{\prime}$ of $\mathrm{Pt}(111)$ in different Pt-water-vacuum setups. Upon adsorption of water on pristine Pt(111), the work function changes with $\Delta \Phi=\Phi_{\mathrm{Pt}(11)}^{\prime}-\Phi_{\mathrm{Pt}(111)} . \Delta \Phi$ is conveniently separated into two contributions: (i) $\Delta \Phi_{\text {orient }}$, given by the static dipole of the isolated water film, corresponding to an expected nominal dipole moment $D_{\mathrm{WL}}=x \mathrm{~d}$; and (ii) $\Delta \Phi_{\text {polar }}$, a polarization contribution, arising due to charge transfer between the water film and the metal surface when they are brought in close contact. $\Delta \Phi_{\text {polar }}$ is strongly dependent on the distance $d_{\mathrm{Pt}-\mathrm{O}}$ between the Pt surface and the $\mathrm{O}$ closest to the metal surface in the first water layer.

\begin{tabular}{|c|c|c|c|c|c|c|}
\hline $\begin{array}{l}\text { Pt-water } \\
\text { model }\end{array}$ & $D_{\mathrm{WL}}$ & $\begin{array}{c}\Phi_{\mathrm{Pt}(111)}^{\prime} \\
(\mathrm{eV})\end{array}$ & $\begin{array}{l}\Delta \Phi \\
(\mathrm{eV})\end{array}$ & $\begin{array}{c}\Delta \Phi_{\text {orient }} \\
(\mathrm{eV})\end{array}$ & $\begin{array}{c}\Delta \Phi_{\text {polar }} \\
(\mathrm{eV})\end{array}$ & $\begin{array}{c}d_{\mathrm{Pt}-\mathrm{O}} \\
(\AA)\end{array}$ \\
\hline$\vec{\rightarrow}$ & $2 d$ & $4.01^{\mathrm{a}} / 4.12^{\mathrm{b}}$ & $-1.59^{\mathrm{a}} /-1.62^{\mathrm{b}}$ & $-1.49^{\mathrm{a}} /-1.35^{\mathrm{b}}$ & $-0.11^{\mathrm{ad}} /-0.26^{\mathrm{bd}}$ & $3.77^{\mathrm{a}} / 3.49^{\mathrm{b}}$ \\
\hline$\vec{a} \vec{\rightarrow}$ & $2 d$ & $3.58^{\mathrm{a}} / 2.91^{\mathrm{b}}$ & $-2.02^{\mathrm{a}} /-2.83^{\mathrm{b}}$ & $-2.08^{\mathrm{a}} /-2.06^{\mathrm{b}}$ & $+0.08^{\mathrm{ad}} /-0.76^{\mathrm{bc}}$ & $3.62^{\mathrm{a}} / 2.72^{\mathrm{b}}$ \\
\hline$\vec{\leftarrow} \rightarrow$ & Od & $5.31^{\mathrm{a}} / 4.43^{\mathrm{b}}$ & $-0.29^{\mathrm{a}} /-1.31^{\mathrm{b}}$ & $-0.12^{\mathrm{a}} /-0.34^{\mathrm{b}}$ & $-0.16^{\mathrm{ad}} /-0.96^{\mathrm{bc}}$ & $3.94^{\mathrm{a}} / 2.33^{\mathrm{b}}$ \\
\hline 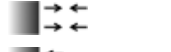 & Od & $5.52^{\mathrm{a}} / 5.41^{\mathrm{b}}$ & $-0.08^{\mathrm{a}} /-0.33^{\mathrm{b}}$ & $-0.02^{\mathrm{a}} /-0.25^{\mathrm{b}}$ & $-0.04^{\mathrm{ad}} /-0.08^{\mathrm{bd}}$ & $4.33^{\mathrm{a}} / 4.17^{\mathrm{b}}$ \\
\hline$\stackrel{\leftarrow}{\rightarrow}$ & Od & $5.43^{\mathrm{a}} / 4.98^{\mathrm{b}}$ & $-0.17^{\mathrm{a}} /-0.76^{\mathrm{b}}$ & $-0.05^{\mathrm{a}} /-0.29^{\mathrm{b}}$ & $-0.11^{\mathrm{ad}} /-0.47^{\mathrm{bc}}$ & $3.74^{\mathrm{a}} / 3.18^{\mathrm{b}}$ \\
\hline$\stackrel{\leftarrow}{\rightarrow}$ & Od & $5.55^{\mathrm{a}} / 4.54^{\mathrm{b}}$ & $-0.05^{\mathrm{a}} /-1.20^{\mathrm{b}}$ & $-0.01^{\mathrm{a}} /-0.22^{\mathrm{b}}$ & $-0.05^{\mathrm{ad}} /-0.97^{\mathrm{bc}}$ & $3.90^{\mathrm{a}} / 2.39^{\mathrm{b}}$ \\
\hline$\stackrel{\leftarrow}{\rightarrow \leftarrow}$ & Od & $5.46^{\mathrm{a}} / 4.65^{\mathrm{b}}$ & $-0.14^{\mathrm{a}} /-1.09^{\mathrm{b}}$ & $-0.05^{\mathrm{a}} /-0.15^{\mathrm{b}}$ & $-0.08^{\mathrm{ad}} /-0.94^{\mathrm{bc}}$ & $3.83^{\mathrm{a}} / 2.44^{\mathrm{b}}$ \\
\hline$\underset{\rightarrow \leftarrow}{\leftarrow}$ & Od & $5.55^{\mathrm{a}} / 5.78^{\mathrm{b}}$ & $-0.05^{\mathrm{a}} /+0.04^{\mathrm{b}}$ & $-0.01^{\mathrm{a}} / 0.05^{\mathrm{b}}$ & $-0.06^{\mathrm{ad}} /-0.05^{\mathrm{bd}}$ & $3.88^{\mathrm{a}} / 4.01^{\mathrm{b}}$ \\
\hline$\vec{a} \stackrel{\leftrightarrow}{\leftrightarrow} \rightarrow \leftarrow$ & $0 \mathrm{~d}$ & $5.44^{\mathrm{a}} / 5.64^{\mathrm{b}}$ & $-0.16^{\mathrm{a}} /-0.10^{\mathrm{b}}$ & $-0.03^{\mathrm{a}} /-0.04^{\mathrm{b}}$ & $-0.12^{\mathrm{ad}} /-0.07^{\mathrm{bd}}$ & $3.72^{\mathrm{a}} / 3.97^{\mathrm{b}}$ \\
\hline$\stackrel{\leftrightarrow}{\leftrightarrow \leftarrow \leftarrow} \rightarrow \leftarrow$ & $-2 \mathrm{~d}$ & $6.54^{\mathrm{a}} / 6.71^{\mathrm{b}}$ & $+0.94^{\mathrm{a}} /+0.97^{\mathrm{b}}$ & $+2.07^{\mathrm{a}} /+2.36^{\mathrm{b}}$ & $-1.12^{\mathrm{ac}} /-1.40^{\mathrm{bc}}$ & $4.09^{\mathrm{a}} / 4.08^{\mathrm{b}}$ \\
\hline 15 & $-2 d$ & $6.73^{\mathrm{a}} / 5.96^{\mathrm{b}}$ & $+1.13^{\mathrm{a}} /+0.22^{\mathrm{b}}$ & $+1.25^{\mathrm{a}} /+0.98^{\mathrm{b}}$ & $-0.06^{\mathrm{ad}} /-0.77^{\mathrm{bc}}$ & $4.10^{\mathrm{a}} / 3.12^{\mathrm{b}}$ \\
\hline$\vec{\leftarrow}$ & $-4 d$ & $7.42^{\mathrm{a}} / 7.63^{\mathrm{b}}$ & $+1.82^{\mathrm{a}} /+1.89^{\mathrm{b}}$ & $+4.24^{\mathrm{a}} /+4.65^{\mathrm{b}}$ & $-2.41^{\mathrm{ac}} /-2.77^{\mathrm{bc}}$ & $4.06^{\mathrm{a}} / 3.80^{\mathrm{b}}$ \\
\hline $1 \pm$ & $-6 \mathrm{~d}$ & $7.69^{\mathrm{a}} / 7.67^{\mathrm{b}}$ & $+2.09^{\mathrm{a}} /+1.93^{\mathrm{b}}$ & $+5.78^{\mathrm{a}} /+5.83^{\mathrm{b}}$ & $-3.69^{\mathrm{ac}} /-3.90^{\mathrm{bc}}$ & $3.83^{\mathrm{a}} / 3.91^{\mathrm{b}}$ \\
\hline 15 & $-8 \mathrm{~d}$ & $7.79^{\mathrm{a}} / 7.82^{\mathrm{b}}$ & $+2.19^{\mathrm{a}} /+2.08^{\mathrm{b}}$ & $+5.61^{\mathrm{a}} /+5.75^{\mathrm{b}}$ & $-3.44^{\mathrm{ac}} /-3.69^{\mathrm{bc}}$ & $3.83^{\mathrm{a}} / 3.86^{\mathrm{b}}$ \\
\hline
\end{tabular}

${ }^{\mathrm{a}} \mathrm{RPBE}$ exchange-correlation functional.

${ }^{\mathrm{b}} \mathrm{PBE}$ exchange-correlation functional.

${ }^{\mathrm{c}}$ Considerable charge transfer between the Pt surface and the water film.

${ }^{\mathrm{d}}$ Small charge transfer between the Pt surface and the water film.

localized at the interface (cf. Fig. 3), but a smaller long-range electron polarization effect that can extend far out in thick water films can also be discerned. ${ }^{53}$ The polarization part is given by

$$
\Delta \Phi_{\text {polar }}=-\frac{e d_{\text {polar }}}{A \varepsilon_{0}},
$$

where $A$ is the surface area of the slab supercell and the net dipole moment due to charge redistribution $d_{\text {polar }}$ is obtained by performing an integration

$$
d_{\text {polar }}=\int_{S} \int_{z_{0}}^{z_{1}} d x d y d z \Delta \rho(x, y, z) z
$$

over the electronic charge density change upon adsorption $\Delta \rho(x, y, z)$. The integration $\int_{S}$ stretches over the surface area $A$ of the slab and the integration $\int_{z_{0}}^{z_{1}}$ starts at the midpoint between two layers in the slab and ends at the center of the vacuum. As seen in Table I, the two contributions $\left(\Delta \Phi_{\text {orient }}+\right.$ $\Delta \Phi_{\text {polar }}$ ) add up to almost exactly $\Delta \Phi$.

When taking a closer look at Table I, we first notice that the net dipole moment of the water film is of paramount importance for the magnitude of the WF. As a rule of thumb, a net dipole moment pointing away from the surface lowers $\Phi_{\operatorname{Pt}(111)}^{\prime}$, whereas the opposite effect is observed for a net dipole moment pointing toward the surface. This result is what you would intuitively expect to see and is fully consistent with earlier findings. ${ }^{27} \mathrm{We}$ also see that when the net dipole starts building up in one direction, that is, when the dipoles of the individual water molecules become more and more oriented in one direction, $\Phi_{\operatorname{Pt}(111)}^{\prime}$ will gradually approach the saturation limit. This effect is clearly demonstrated also in Fig. 2(b) where up to three H-down bilayers have been stacked upon each other. Further inspection of Table I reveals that the magnitude of $\Delta \Phi_{\text {polar }}$ is strongly correlated with the distance $d_{\mathrm{Pt}-\mathrm{O}}$ between the Pt surface and the $\mathrm{O}$ closest to the metal surface in the first water layer, especially for systems with moderate charge redistribution $\left(\left|\Delta \Phi_{\text {polar }}\right| \leqslant 1\right)$. Moreover, the RPBE and PBE functionals often give substantially different $\Phi_{\mathrm{Pt}(111)}^{\prime}$. This difference can also be traced back to variations in $\Delta \Phi_{\text {polar }}$. PBE usually predicts a smaller metal-water separation, resulting in a larger charge redistribution. If, on the other hand, the electronic structure is calculated for a fixed nuclear geometry, the two functionals yield very similar $\Phi_{\mathrm{Pt}(111)}^{\prime}$.

It is worth noticing that, even when performing calculations at the PBE level, we generally overestimate the metal-water separation for H-down (3.12 $\AA$ ) and H-up (3.49 $\AA$ ) structures compared to corresponding theoretical values found in the literature. Other authors typically report $d_{\mathrm{Pt}-\mathrm{O}} \mathrm{S}$ of approximately $2.7 \AA$ for these structures. ${ }^{11,27,43,44,49}$ As a result, compared to others, we underestimate the charge transfer and, thus, get a less negative $\Delta \Phi_{\text {polar }}$ and a larger $\Phi_{\mathrm{Pt}(111)}^{\prime}$. Most experimental values reported for the PZC of $\mathrm{Pt}$ are of the order $0.4 \mathrm{~V}$ versus $\mathrm{SHE},{ }^{31,54-56}$ which would correspond to a $\Phi_{\mathrm{Pt}(111)}^{\prime}$ of about $4.8 \mathrm{eV}$ and a water-induced WF change of approximately $-1 \mathrm{eV}$. Obviously, among our systems, only a few of the neutral water films, treated at the PBE level, match these experimental values. We note, however, as pointed out before, ${ }^{6}$ that a really rigorous treatment of the PZC would require statistical averaging, either over a complete set of static low-temperature water models, weighted by their total energy 

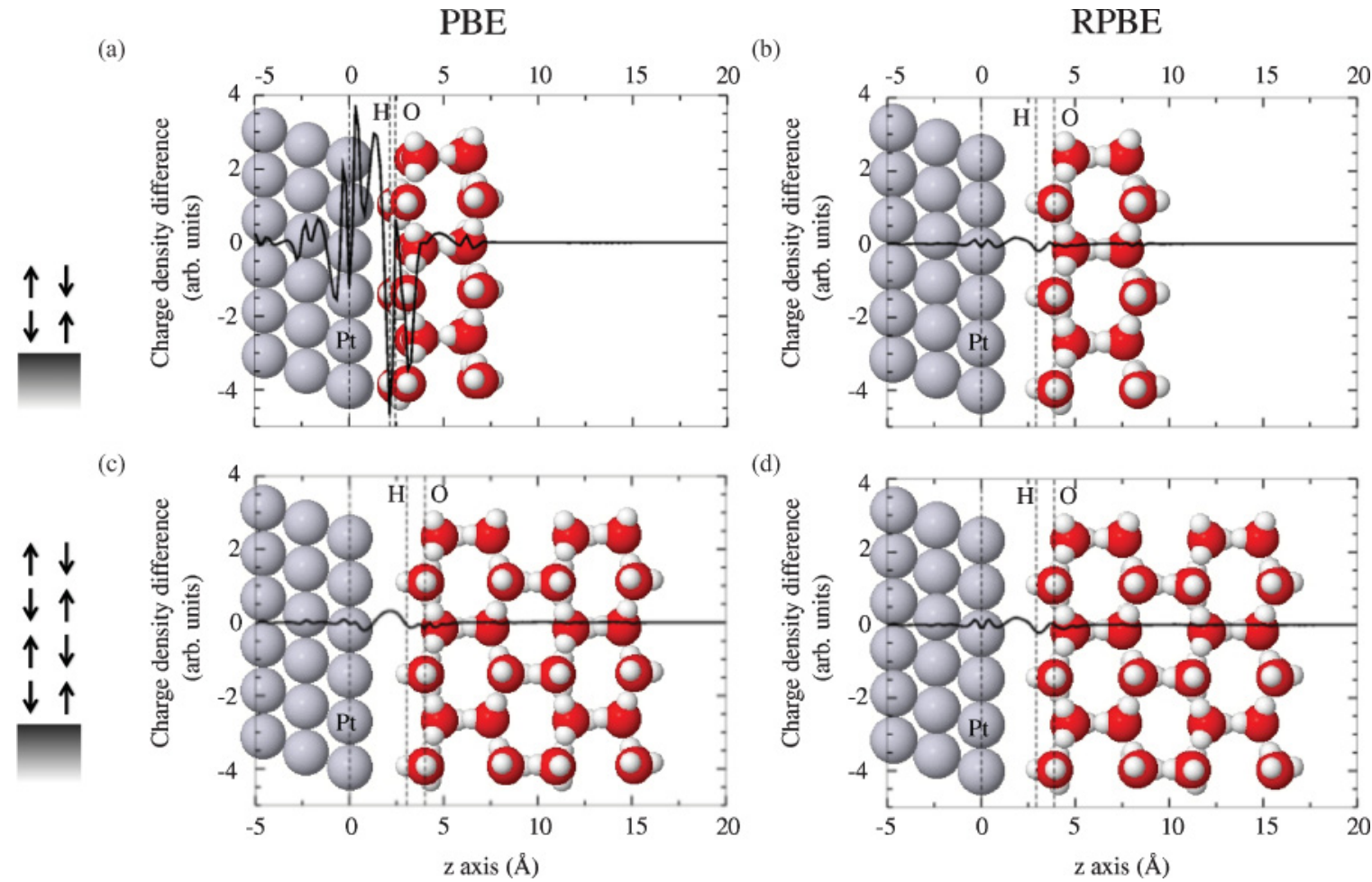

(d)

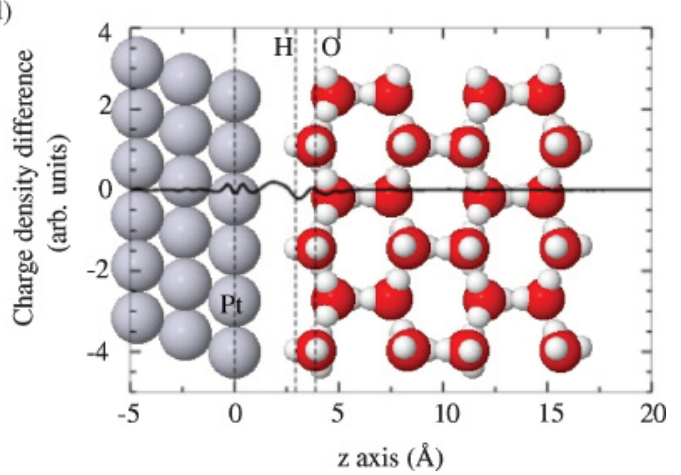

FIG. 3. (Color online) Optimized structures of two [(a), (b)] and four [(c), (d)] H-mixed water bilayers adsorbed on Pt(111). The calculations were performed with the PBE [(a), (c)] and RPBE [(b), (d)] XC functionals. The solid black lines indicate laterally averaged redistribution of electronic charge upon adsorption of the water films.

(stability), or of a long room-temperature molecular dynamics simulation.

To gain further insight into the difference between the two $\mathrm{XC}$ functionals and the relationship between the metal-water separation and the WF, we analyze the stacks of H-mixed bilayers more carefully. Special attention is paid to how the geometry and the WF change as the thickness of the water film is increased. Figure 3 shows the $0-\mathrm{K}$ structures of $\mathrm{Pt}(111)$ with water films consisting of two and four H-mixed layers, optimized with PBE and RPBE, respectively. For the thinner film, PBE predicts a much smaller Pt-water distance $\left(d_{\mathrm{Pt}-\mathrm{O}}=2.39 \AA\right)$ than $\operatorname{RPBE}\left(d_{\mathrm{Pt}-\mathrm{O}}=3.90 \AA\right)$. The smaller separation results in a much larger charge transfer (cf. Table I and Fig. 3) and, hence, a much larger contribution from $\Delta \Phi_{\text {polar }}$ to $\Phi_{\operatorname{Pt}(111)}^{\prime}$. For this particular system, PBE predicts a $1-\mathrm{eV}$ smaller $\Phi_{\mathrm{Pt}(111)}^{\prime}$ than RPBE, mainly due to the charge-transfer effect. When more water layers are added, little happens to the equilibrium distance in the RPBE simulation $\left(d_{\mathrm{Pt}-\mathrm{O}}=3.88 \AA\right.$ for the four-layer-thick film) and, hence, $\Phi_{\mathrm{Pt}(111)}^{\prime}$ remains fairly constant. However, with PBE, the separation suddenly increases to $d_{\mathrm{Pt}-\mathrm{O}}=4.01 \AA$ as the fourth layer is added. Accordingly, the amount of charge transferred is now similar to that predicted by RPBE (cf. Fig. 3) and $\Phi_{\mathrm{Pt}(111)}^{\prime}=5.78 \mathrm{eV}$ also agrees well with the $\Phi_{\mathrm{Pt}(111)}^{\prime}=5.55 \mathrm{eV}$ given by RPBE. A similar trend is observed for stacks of H-down bilayers; in the RPBE case, $d_{\mathrm{Pt}-\mathrm{O}}$ remains almost constant, whereas for PBE, it jumps by $\sim 0.7 \AA$ after adding the second bilayer (cf. Table I).

The abrupt change in $d_{\mathrm{Pt}-\mathrm{O}}$, in the case of PBE, could be a manifestation of a competition between hydrogen-bondmediated interlayer interactions in the water film on one hand and the film's desire to minimize its surface energy at the interface on the other. ${ }^{57}$ The two competing contributions would assume optimal values at different points in geometry space. When the fourth layer is added, the interlayer interaction starts to dominate, resulting in a change in $d_{\mathrm{Pt}-\mathrm{O}}$. In the case of RPBE, the metal-water interaction is much weaker, and the inter-layer interaction will dominate already from the beginning. In connection with this discussion, it should be noted that the opposite trend, i.e., a reduction of $d_{\mathrm{Pt}-\mathrm{O}}$ with the number of water layers, has been observed for stacks of H-up bilayers. ${ }^{44}$ At the same time, it is also worth mentioning that careful analysis of literature data has indicated that the magnitude of $\Delta \Phi$ determined from water adsorption data is most likely higher than the corresponding $\Delta \Phi$ at the electrochemical interface, where the thickness of the water reaches macroscopic dimensions. ${ }^{31}$ If this observation is true, it is consistent with our finding of an increase in $d_{\mathrm{Pt}-\mathrm{O}}$ and reduction in charge transfer for thicker films. Hence, whether the relatively large $d_{\mathrm{Pt}-\mathrm{O}}$ we observe for thick films composed of H-mixed bilayers represents a true physical property of the interface or if it is a computational artifact is uncertain, especially given current density functionals' limited ability to accurately estimate the energy of hydrogen bonds.

\section{B. Trends in the PZC and sensitivity of the ASHEP to water structure}

For high-quality calculations of the ASHEP, one should demand from the water film representing the electrolyte (i) that it exhibits no net dipole moment when uncharged, 
(ii) that it exchanges no or very little charge with the adjacent metal under PZC conditions, and (iii) that its water network is flexible. The first criterion simply ensures that the model reproduces the expected zero average net dipole of a thick finite-temperature water film. Imposing the second criterion guarantees that additional hydrogen atoms added to the water film will donate electrons to the metal, which results in a decrease of the electrode potential. Although experimental measurements of water-induced WF changes suggest that some charge is indeed exchanged between the water film and the metal at the PZC, we would like this charge transfer to be small for computational reasons. If the charge transfer would be significant already at the PZC, one could in the extreme case end up in a situation where additional hydrogen atoms will not donate any charge to the metal surface. Then, it would no longer be possible to construct the free-energy parabolae, discussed in Sec. IV, on which our analysis of the ASHEP is based. Finally, the third criterion is to ensure that the film, just like bulk water with its high dielectric constant, is efficient at screening electric fields.

Out of the manifold structures used in the analysis of the PZC, we have selected four (see Fig. 4) that fulfill a varying number of the above criteria, for further investigation. Their properties are qualitatively different in terms of net dipole, charge transfer, and ability to screen electric fields. We calculate the ASHEP and PZC, at the RPBE level, on the eight close-packed transition-metal surfaces using these water structures. The subsequent analysis of the results then provides a clear indication how and to what degree the various criteria influence the theoretical ASHEP. More precisely, the water models chosen include the H-mixed/H-mixed structure (model 1), a flexible water structure, which, at the RPBE level, is predicted to exhibit negligible charge transfer and net dipole at the PZC; the H-down structure (model 2) with no charge transfer but finite dipole moment; the $\mathrm{H}$-up/H-down structure (model 3), a more rigid water structure with essentially zero charge transfer and net dipole; and, finally, the $\mathrm{H}$-down/Hmixed structure (model 4) with finite dipole and substantial charge transfer. How well the four water models satisfy the suggested physical criteria can be seen in Table. II. Aside from the aspiration for diversity in electronic properties, the (a)

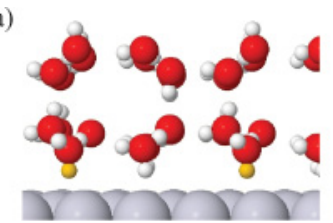

(c)

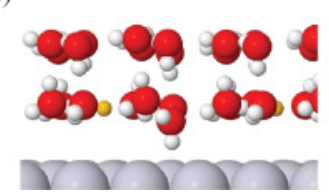

(b)

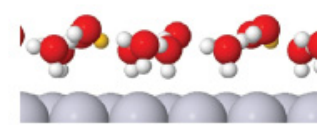

(d)

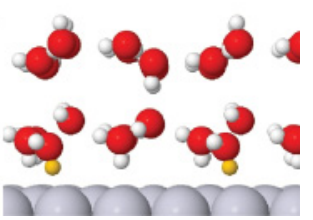

FIG. 4. (Color online) Structures of four different water models, each containing 1 additional hydrogen atom per 12 surface metal atoms. (a) Model 1: H-mixed/H-mixed structure. (b) Model 2: Hdown structure. (c) Model 3: H-up/H-down structure. (d) Model 4: H-down/H-mixed structure.
TABLE II. Checkboard showing to what extent each of the four tested water models satisfy the criteria that have to be fulfilled under PZC conditions in order to ensure a correct estimate of the ASHEP.

\begin{tabular}{lccc}
\hline $\begin{array}{l}\text { Water } \\
\text { model }\end{array}$ & $\begin{array}{c}\text { No net } \\
\text { dipole }\end{array}$ & $\begin{array}{c}\text { No charge } \\
\text { transfer }\end{array}$ & $\begin{array}{c}\text { Flexibility of } \\
\text { water network }\end{array}$ \\
\hline Model 1 & $\mathrm{x}$ & $\mathrm{x}$ & $\mathrm{x}$ \\
Model 2 & $\mathrm{x}$ & $\mathrm{x}$ & \\
Model 3 & $\mathrm{x}$ & & $\mathrm{x}$ \\
Model 4 & & & \\
\hline \hline
\end{tabular}

choice of water models was stipulated by computational cost (the water films should contain as few water molecules as possible) and the possibility of adding hydrogen atoms to the water structure without disrupting it (i.e., without rearranging the water dipoles). The latter constraint is the reason why out of the three possible mono-bilayer structures (H-down, H-up, and H-mixed), only the H-down structure was selected; the dipoles pointing up in the other two models have a strong tendency to reorient toward the surface after addition of extra hydrogen atoms.

To determine the ASHEP, we use the scheme for coupling the work function scale to the thermochemical scale, outlined in Sec. IV and described in detail in our previous works. ${ }^{15,16}$ Each individual $G_{\text {int }}$-versus- $\Phi^{\prime}$ parabola, used to establish a link between the two scales for one specific system, contains a point corresponding to the PZC (cf. Fig. 6, Appendix B). Hence, for each metal and water model, we automatically obtain the PZC as part of the ASHEP calculation.

In Fig. 5, we have plotted the absolute values of the potential of zero charge and standard hydrogen electrode ( $U_{\mathrm{PZC}}$ and $U_{\mathrm{SHE}}$, obtained from the parabolas in Appendix B), for the eight close-packed transition metals, against the work function $\Phi$ of the corresponding pristine metal surfaces. From these plots, it is evident that the choice of water model will affect the calculated value of both potentials. The PZC results are consistent with the previous finding for $\operatorname{Pt}(111)$ that the value of $U_{\mathrm{PZC}}$ is dictated by the water structure, which complicates comparison with experimental $U_{\text {PZC }}$ data and limits the conclusions that can be drawn from calculations on one specific metal-water system. Yet, since we apply each water model to a range of metals, it is still possible to discern some general trends. For instance, $U_{\mathrm{PZC}}$ is found to vary linearly with $\Phi$. Such linear relations between $U_{\mathrm{PZC}}$ and $\Phi$ have been observed before, in measurements on different facets of Au and $\mathrm{Ag},{ }^{30}$ and was theoretically predicted by Bockris ${ }^{34}$ who emphasized that the linearity will depend upon whether the water dipoles or charge transfer is independent of the nature of the metals. In other words, the linear trend is expected to hold as long as the water structure of the interface is fairly constant, i.e., if the dipoles assume the same orientation on all metals, which is exactly the case for our artificially constructed water films. When the charge transfer is negligible, as in models 1-3, the slope will be close to 1 [cf. Figs. 5(a)-5(c)]. On the other hand, when the charge transfer is substantial as in model 4 , the slope becomes essentially zero. This is due to the fact that the water at the interface then acts as a perfect screening medium. Accordingly, the potential drop at the interface vanishes and 

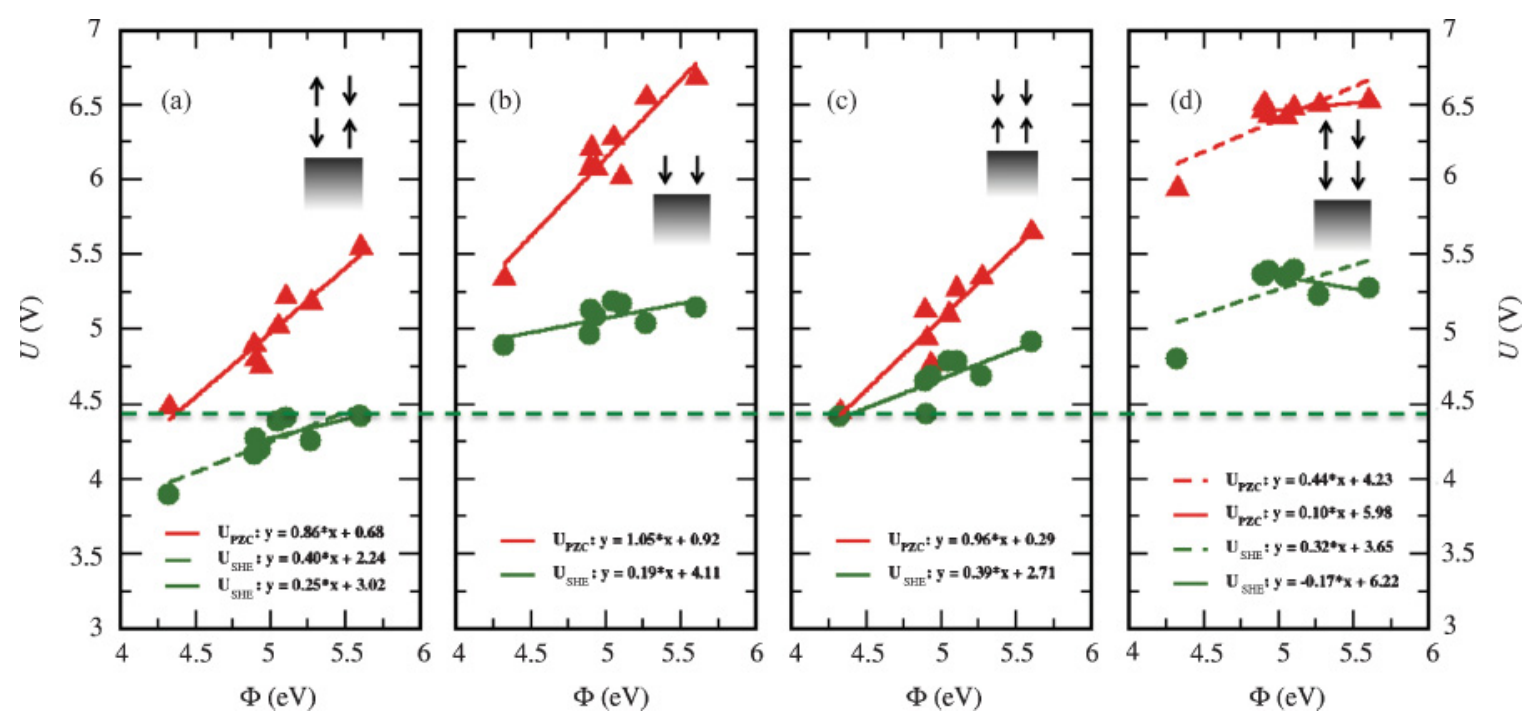

FIG. 5. (Color online) Dependence of the potential of zero charge (red, triangles) and the potential of the standard hydrogen electrode (green, circles) on the pristine metal work function for (a) model 1, (b) model 2, (c) model 3, and (d) model 4. The horizontal dashed line indicates the experimental reference value $(4.44 \mathrm{~V})$ for the absolute standard hydrogen electrode potential. Notice that the point with the lowest work function $(\mathrm{Ag})$ is off in models 1 and 4 because of the instability of the water film in these systems. The dashed and solid lines are the fits with and without this point.

the WF will be solely determined by the surface dipole at the water-vacuum interface.

All the differences pertaining to $U_{\mathrm{PZC}}$ should, in principle, be eliminated under SHE conditions, since SHE is the universal reference point and, as such, independent of the electrode material used in the measurements (see the discussion in Sec. II). Therefore, ideally, the slope of $U_{\text {SHE }}(\Phi)$ should be zero. A $U_{\mathrm{SHE}}(\Phi)$ slope other than zero thus reflects the imperfect screening of the water used in the simulation. As evident from Fig. 5, model 4 possesses the best and model 3 the worst screening properties. This result can be rationalized in terms of the ability of water layers to adjust their position and the amount of charge transferred. When water molecules in the first layer point toward the surface, they will be more effective in screening than in the case when they are unphysically constrained and form a rather rigid water structure as in model 3.

One can further conclude from Fig. 5 that the magnitude of the ASHEP and, thus, its deviation from the experimental reference $(4.44 \mathrm{~V})$ depends mainly on the net dipole of the water film, but also to some extent on the film's ability to facilitate charge transfer. The ASHEP is commonly measured in bulk solution, not at the metal-water interface as in our approximate models. Therefore, a water film featuring no net dipole is a better representation of the experimental situation and, consequently, models 1 and 3 exhibit ASHEP values closest to the experimental benchmark. The fact that the average ASHEP values predicted by models 1 and 3 [4.25 (4.30 without $\mathrm{Ag}$ ) and $4.68 \mathrm{~V}$ ] are in rather good agreement with the experimental value may seem surprising given the significant overestimation of PZC (cf. Table I and the discussion in Sec. V A). However, the additional protons present in the first water bilayer at potentials cathodic of PZC will help reduce the metal-water separation significantly. Hence, as we start charging the surface, the separation will soon decrease to a value that would be more consistent with the distance in the real system under PZC conditions. As the surface is further charged, the separation does not change much. Accordingly, the points further to the left on the $G_{\text {int }}$-versus- $\Phi^{\prime}$ parabolae may in a way correspond to more accurately described interfaces; hence, the surprisingly good estimates.

To conclude, in the beginning of this section, it was suggested that a water film employed in a calculation of the ASHEP should satisfy three physical criteria. We could see in Table II that only model 1 satisfies all three conditions. The ensuing calculations and analysis then confirmed that this model also gives the best estimate of the ASHEP. Indisputably, model 1 predicts too high a value for the PZC when calculations are carried out at the RPBE level. However, despite this overestimation, some interesting general trends can be discerned when the model is applied to a larger set of metals, and, most importantly, for reasons discussed above this error is not reflected in the ASHEP.

\section{Internal versus external potential reference}

Finally, we would like to briefly comment on the use of external and internal ASHEP references. By external reference we mean a universal reference, such as the one we have tried to establish in this study, that is valid for all metals and in good agreement with the experimental counterpart. By internal reference we instead mean a reference that is valid only for one particular metal-water system and can be obtained from a free-energy parabola such as one of those found in Fig. 6 in Appendix B. For some problems, such as the matching of semiconductor and solution energy levels mentioned in the Introduction, the use of an external reference is a necessity. However, for tackling other problems, notably the energetics of charge-transfer reactions, using an internal reference is sufficient. Employing an internal reference often significantly 
(a)

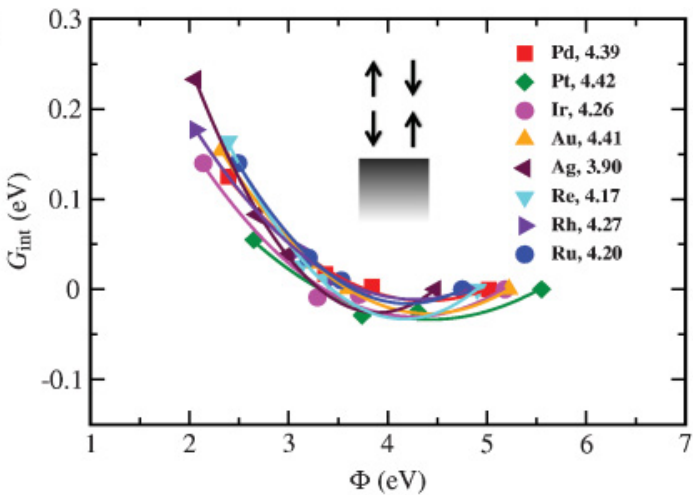

(c)

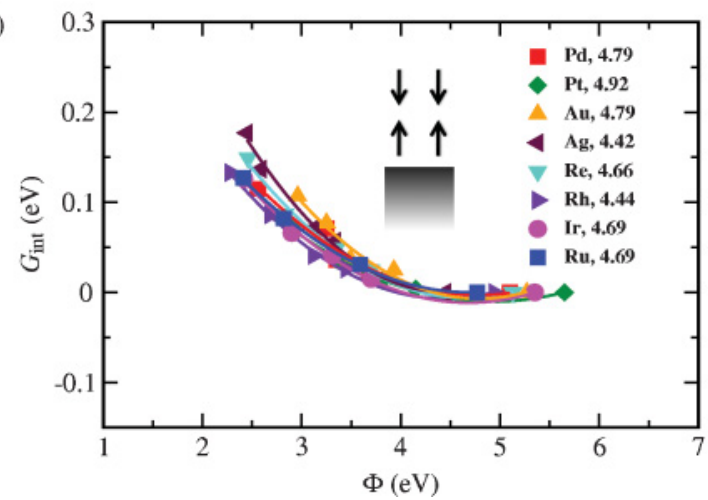

(b)

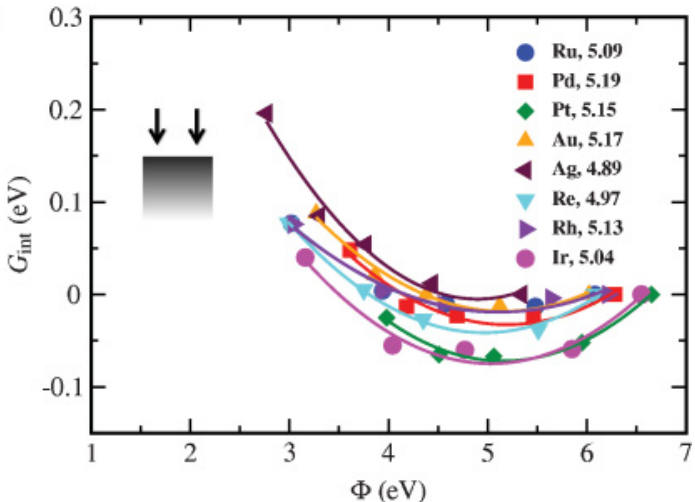

(d)

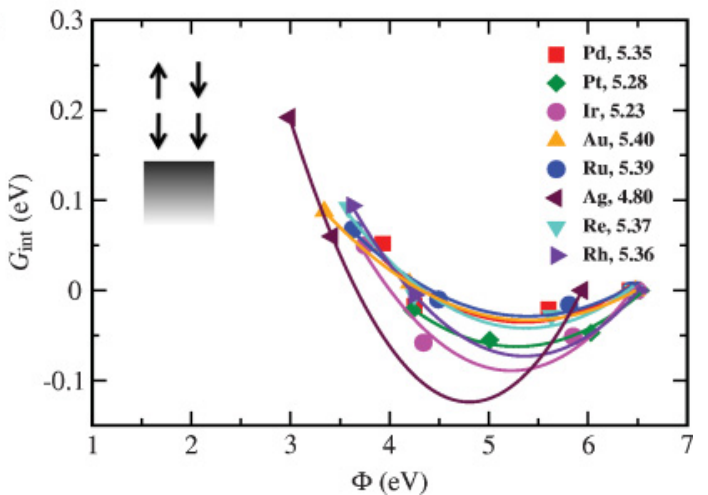

FIG. 6. (Color online) Dependence of the integral free energy $G_{\text {int }}$ on the work function of the metal in contact with water for the eight investigated transition metals, shown for (a) model 1, (b) model 2, (c) model 3, and (d) model 4. The mean average value and standard deviation of the ASHEP are 4.25/0.17 V with Ag and 4.30/0.10 V without Ag for model 1, 5.08/0.11 V for model 2, 4.68/0.17 V for model 3, and 5.27/0.20 V with Ag and 5.34/0.06 V without Ag for model 4.

reduces the computational burden because it allows the use of simpler water models. It also ensures that errors introduced by the use of approximate water structures and different electrode materials will be born out. The latter is a consequence of error cancellations between the $U_{\mathrm{SHE}}$ point and any other point on the same $G_{\text {int }}$-versus- $\Phi^{\prime}$ parabola. Hence, for studies of charge-transfer reactions, we do not expect that the use of the exact water model, capable of accurately screening and solvating the proton, will significantly improve results obtained with simpler models. We have previously ascertained this point by showing that the energetics of the hydrogen evolution-oxidation reactions are preserved, regardless of the interfacial water structure. ${ }^{16}$

\section{SUMMARY AND CONCLUSIONS}

In DFT based models of electrochemical systems, the work function (WF) of the electrode, placed in an electrode-watervacuum environment, is usually used as a measure of the absolute potential. Determining the WF that corresponds to the absolute standard hydrogen electrode potential (ASHEP) with this kind of setup constitutes a great challenge. Due to present limitations in computer power, it is not practically feasible to emulate bulk water in a large-scale electrochemistry study. Instead, one is usually limited to much less sophisticated water models. The near-vacuum reference level for the electrons in such models is directly dependent on the structure of the water layer, thus affecting the calculated WF. This uncertainty translates into an arbitrariness not only in the calculated ASHEP, but also in the theoretical estimate of the potential of zero charge (PZC) of an electrode. In this paper, we have made an attempt to shed some light on these issues. The sensitivity of the PZC to water structure has been quantified through detailed analysis of a large set of $\mathrm{Pt}(111)$-water systems. Likewise, by systematically analyzing four different water structures on a series of close-packed transition-metal surfaces, we have demonstrated how the choice of water model affects the calculated ASHEP. However, to properly assess and compare the qualities of ASHEPs obtained with different water models, one needs a well-grounded experimental benchmark. After careful examination of the available literature, it was concluded that the most reliable value of those measured using experimental setups matching our DFT model is $4.44 \mathrm{~V}$.

To quantify the effect of water structure on the calculated $\mathrm{PZC}$, we split the change in $\mathrm{WF}$ of $\mathrm{Pt}(111)$ resulting from adsorption of a water film into two terms: a static dipole term, determined by the orientation of the water molecules, and a charge polarization term, reflecting the amount of charge transferred between the water and the metal. In general, both terms contributed significantly to the total change in WF and the size of the latter was found, not surprisingly, to correlate strongly with the distance between the metal and the first water bilayer. Furthermore, we investigated what impact the choice of exchange correlation (XC) functional, PBE or RPBE, has on the results. A significant difference was observed between $P B E$ and RPBE for systems containing only a few water bilayers 
when the first bilayer contained molecular dipoles oriented toward the surface. PBE consistently gave lower WF estimates. This corresponds to a lower PZC and implicitly influences also the ASHEP, although probably to a somewhat lesser extent. The origin of the lower WFs in the PBE calculations could be traced back to smaller metal-water separations, something that promotes charge transfer. However, as more water layers were added, the metal-water separation suddenly increased in the PBE calculation to a distance similar to that predicted by RPBE. As a consequence, the difference in WF vanished at this point.

Finally, based partly on the PZC study, we recognized three properties that the model water film must possess in order to yield an ASHEP value that is close to the experimental benchmark and independent of the metal surface; under PZC conditions, the film (i) should have no net dipole moment, (ii) should not facilitate charge transfer to the metal surface, and (iii) should consist of a water network that is flexible enough to allow efficient screening. We then demonstrated the importance of these criteria by evaluating the ASHEP for four simple, but qualitatively different, water structures. A water film consisting of two neutral water bilayers stacked upon each other was identified as the best choice. It appeared to possess all three properties and produced the ASHEP closest to the experimental benchmark.

\section{ACKNOWLEDGMENTS}

CAMD is funded by the Lundbeck foundation. The Catalysis for Sustainable Energy initiative is funded by the Danish Ministry of Science, Technology and Innovation. Support from the Icelandic Research Foundation, the Danish Center for Scientific Computing, the Danish Council for Technology and Innovation's FTP program, and the Strategic Electrochemistry Research Center is gratefully acknowledged.

\section{APPENDIX A: COMPUTATIONAL DETAILS}

The electronic-structure calculations have been carried out using density functional theory with the Perdew-BurkeErnzerhof $^{58}$ (PBE) and revised Perdew-Burke-Ernzerhof ${ }^{59}$ (RPBE) functionals for exchange and correlation. Lattice constants were optimized for bulk metals using the RPBE functional and were then used in all calculations, including those with the PBE functional. Metal electrodes were represented by periodically repeated three layer slabs, separated by at least $12 \AA$ of vacuum in the direction perpendicular to the surface. This amount of vacuum ensured convergence of work functions and energies. Inclusion of a fourth layer had negligible influence on the presented results. Surface unit cells of various sizes, $(3 \times 2),(3 \times 3),(3 \times 4),(6 \times 3)$ and $(6 \times 4)$, sampled with $(4 \times 6),(4 \times 4),(4 \times 3),(2 \times 4)$, and $(2 \times 3)$ Monkhorst-Pack k-point sampling grids, ${ }^{60}$ were used to account for different proton concentrations (potentials). In all cases, symmetry was applied to further reduce the number of $\mathrm{k}$ points. The dipole correction was used in all cases to decouple the electrostatic interaction between the periodically repeated slabs. ${ }^{61}$ The Kohn-Sham equations were solved using a plane-wave basis set with a plane wave and density cutoff of 26 Ry. Ionic cores were described with Vanderbilt ultrasoft pseudopotentials. ${ }^{62}$ A Fermi smearing of $0.1 \mathrm{eV}$ was used, and energies were extrapolated to an electronic temperature of $0 \mathrm{~K}$. The two bottom layers of the slab were fixed in their bulk positions, while all other atoms were relaxed until the magnitude of the forces acting on them were less than $0.01 \mathrm{eV} / \AA$. All calculations were performed using the DACAPO code, ${ }^{63}$ integrated with the atomic simulation environment. ${ }^{64}$

\section{APPENDIX B: INTEGRAL FREE ENERGY}

In Fig. 6, the integral free energy $G_{\text {int }}$ obtained with models $1,2,3$, and 4 , respectively, has been plotted versus the WF $\Phi^{\prime}$ calculated for the metal-water-vacuum setup. The points $G_{\text {int }}=0$ to the far right in the graphs are obtained for the uncharged systems, without any additional hydrogen in the water, and thus correspond to the $U_{\mathrm{PZC}}$ of the different metals. The minima of the parabolae, on the other hand, correspond to the $U_{\mathrm{SHE}} \mathrm{S}$ [cf. Eq. (3)]. The points left of $U_{\mathrm{PZC}}$, i.e., of lower potential (higher proton concentration), have been obtained by adding a single hydrogen atom to unit cells of varying size $[(3 \times 2),(3 \times 4),(6 \times 3),(6 \times 4)]$. The most stable position for the additional hydrogen is in the first bilayer, coordinated to a planar water molecule and pointing down to the metal surface. Each simulation cell provides several such positions, but they are all geometrically and energetically equivalent, which means that the parabolae are uniquely defined. Notice that only three points have been used to define the $\mathrm{Au}, \mathrm{Ag}$, and $\mathrm{Rh}$ parabolae in model 4 . Including more points would improve the accuracy, but unfortunately points corresponding to large surface cells could not be obtained due to substantial reconstruction of the water.

\footnotetext{
*jross@fysik.dtu.dk

${ }^{1}$ S. Trasatti, Pure Appl. Chem. 58, 955 (1986).

${ }^{2}$ Y. Cai and A. B. Anderson, J. Phys. Chem. B 108, 9829 (2004).

${ }^{3}$ Y. Cai, A. B. Anderson, J. C. Angus, and L. N. Kostadinov, Electrochem. Solid-State Lett. 8, E62 (2005).

${ }^{4}$ M. Otani and O. Sugino, Phys. Rev. B 73, 115407 (2006).

${ }^{5}$ J.-S. Filhol and M. Neurock, Angew. Chem., Int. Ed. 45, 402 (2006).

${ }^{6}$ C. D. Taylor, S. A. Wasileski, J.-S. Filhol, and M. Neurock, Phys. Rev. B 73, 165402 (2006).

${ }^{7}$ P. H.-L. Sit, M. Cococcioni, and N. Marzari, Phys. Rev. Lett. 97, 028303 (2006).
}

${ }^{8}$ E. Santos, M. T. M. Koper, and W. Schmickler, Chem. Phys. Lett. 419, 421 (2006).

${ }^{9}$ E. Santos, P. Quaino, and W. Schmickler, Angew. Chem., Int. Ed. 46, 8262 (2007).

${ }^{10}$ E. Skúlason, G. S. Karlberg, J. Rossmeisl, T. Bligaard, J. Greeley, H. Jónsson, and J. K. Nørskov, Phys Chem Chem Phys 9, 3241 (2007).

${ }^{11}$ R. Jinnouchi and A. B. Anderson, Phys. Rev. B 77, 245417 (2008).

${ }^{12}$ M. J. Janik, C. D. Taylor, and M. Neurock, J. Electrochem. Soc. 156, B126 (2009). 
${ }^{13}$ J. K. Nørskov, J. Rossmeisl, A. Logadottir, L. Lindqvist, J. R. Kitchin, T. Bligaard, and H. Jonsson, J. Phys. Chem. B 108, 17886 (2004).

${ }^{14}$ R. Jinnouchi and A. B. Anderson, J. Phys. Chem. C 112, 8747 (2008).

${ }^{15}$ J. Rossmeisl, E. Skúlason, M. E. Björketun, V. Tripkovic, and J. K. Nørskov, Chem. Phys. Lett. 466, 68 (2008).

${ }^{16}$ E. Skúlason, V. Tripkovic, M. E. Björketun, S. Gudmundsdóttir, G. S. Karlberg, J. Rossmeisl, T. Bligaard, H. Jónsson, and J. K. Nørskov, J. Phys. Chem. C 114, 18182 (2010).

${ }^{17}$ J. E. B. Randles, Trans. Faraday Soc. 52, 1573 (1956).

${ }^{18}$ J. R. Farrell and P. McTigue, J. Electroanal. Chem. Interfacial Electrochem. 139, 37 (1982).

${ }^{19}$ R. Gomer and G. Tryson, J. Chem. Phys. 66, 4413 (1977).

${ }^{20}$ W. N. Hansen and D. M. Kolb, J. Electroanal. Chem. Interfacial Electrochem. 100, 493 (1979).

${ }^{21}$ E. R. Kötz, H. Neff, and K. Müller, J. Electroanal. Chem. Interfacial Electrochem. 215, 331 (1986).

${ }^{22}$ J. Schneider, D. Franke, and D. M. Kolb, Surf. Sci. 198, 277 (1988).

${ }^{23}$ W. N. Hansen and G. J. Hansen, Phys. Rev. A 36, 1396 (1987).

${ }^{24}$ H. Reiss and A. Heller, J. Phys. Chem. 89, 4207 (1985).

${ }^{25}$ W. R. Fawcett, Langmuir 24, 9868 (2008).

${ }^{26}$ A. A. Isse and A. Gennaro, J. Phys. Chem. B 114, 7894 (2010).

${ }^{27}$ S. Schnur and A. Groß, New J. Phys. 11, 125003 (2009).

${ }^{28}$ S. Schnur and A. Groß, Catal. Today 165, 129 (2011).

${ }^{29}$ O. Sugino, I. Hamada, M. Otani, Y. Morikawa, T. Ikeshoji, and Y. Okamoto, Surf. Sci. 601, 5237 (2007).

${ }^{30}$ S. Trasatti, Surf. Sci. 335, 1 (1995).

${ }^{31}$ S. Trasatti, Electrochim. Acta 36, 1659 (1991).

${ }^{32} \mathrm{~S}$. Trasatti and E. Lust, Modern Aspect of Electrochemistry, Number 33 (Kluwer, New York, 1999).

${ }^{33}$ S. Trasatti, J. Electroanal. Chem. Interfacial Electrochem. 139, 1 (1982)

${ }^{34}$ J. O. Bockris and S. D. Argade, J. Chem. Phys. 49, 5133 (1968).

${ }^{35}$ D. Tsiplakides and C. G. Vayenas, J. Electrochem. Soc. 148, E189 (2001).

${ }^{36}$ J. M. Heras and L. Viscido, Catal. Rev. Sci. Eng. 30, 281 (1988).

${ }^{37}$ P. A. Thiel and T. E. Madey, Surf. Sci. Rep. 7, 211 (1987).

${ }^{38}$ M. D. Tissandier, K. A. Cowen, W. Y. Feng, E. Gundlach, M. H. Cohen, A. D. Earhart, and J. V. Coe, J. Phys. Chem. A 102, 7787 (1998).

${ }^{39}$ C. Clay, S. Haq, and A. Hodgson, Phys. Rev. Lett. 92, 046102 (2004).
${ }^{40}$ H. Ogasawara, B. Brena, D. Nordlund, M. Nyberg, A. Pelmenschikov, L. G. M. Pettersson, and A. Nilsson, Phys. Rev. Lett. 89, 276102 (2002).

${ }^{41}$ G. S. Karlberg, F. E. Olsson, M. Persson, and G. Wahnström, J. Chem. Phys. 119, 4865 (2003).

${ }^{42}$ A. Michaelides and P. Hu, J. Chem. Phys. 114, 513 (2001).

${ }^{43}$ S. Meng, L. F. Xu, E. G. Wang, and S. Gao, Phys. Rev. Lett. 89, 176104 (2002)

${ }^{44}$ S. Meng, E. G. Wang, and S. Gao, Phys. Rev. B 69, 195404 (2004).

${ }^{45}$ P. J. Feibelman, Science 295, 99 (2002).

${ }^{46}$ C. Clay, S. Haq, and A. Hodgson, Chem. Phys. Lett. 388, 89 (2004).

${ }^{47}$ C. Clay, L. Cummings, and A. Hodgson, Surf. Sci. 601, 562 (2007).

${ }^{48}$ F. T. Wagner and T. E. Moylan, Surf. Sci. 191, 121 (1987).

${ }^{49}$ S. Nie, P. J. Feibelman, N. C. Bartelt, and K. Thürmer, Phys. Rev. Lett. 105, 026102 (2010).

${ }^{50}$ V. Tripkovic, E. Skúlason, S. Siahrostami, J. K. Nørskov, and J. Rossmeisl, Electrochim. Acta 55, 7975 (2010).

${ }^{51}$ CRC Handbook of Chemistry and Physics, 91st ed. (CRC Press, New York, 2010).

${ }^{52}$ G. Materzanini, G. F. Tantardini, P. J. D. Lindan, and P. Saalfrank, Phys. Rev. B 71, 155414 (2005).

${ }^{53}$ S. Meng, E. G. Wang, and S. Gao, J. Chem. Phys. 119, 7617 (2003).

${ }^{54}$ T. Pajkossy and D. M. Kolb, Electrochim. Acta 46, 3063 (2001).

${ }^{55}$ V. Climent, B. A. Coles, and R. G. Compton, J. Phys. Chem. B 106, 5988 (2002).

${ }^{56}$ E. Langenbach, A. Spitzer, and H. Lüth, Surf. Sci. 147, 179 (1984).

${ }^{57}$ J. Rossmeisl, J. K. Nørskov, and K. W. Jacobsen, J. Am. Chem. Soc. 126, 13140 (2004).

${ }^{58}$ J. P. Perdew, K. Burke, and M. Ernzerhof, Phys. Rev. Lett. 77, 3865 (1996).

${ }^{59}$ B. Hammer, L. B. Hansen, and J. K. Nørskov, Phys. Rev. B 59, 7413 (1999).

${ }^{60}$ H. J. Monkhorst and J. D. Pack, Phys. Rev. B 13, 5188 (1976).

${ }^{61}$ L. Bengtsson, Phys. Rev. B 59, 12301 (1999).

${ }^{62}$ D. Vanderbilt, Phys. Rev. B 41, 7892 (1990).

${ }^{63}$ DACAPO pseudopotential code, [https://wiki.fysik.dtu.dk/dacapo], Center for Atomic-scale Materials Design (CAMD), Technical University of Denmark.

${ }^{64}$ Atomic Simulation Environment (ASE), [https://wiki.fysik.dtu.dk/ ase], Center for Atomic-scale Materials Design (CAMD), Technical University of Denmark. 